\title{
LA PARTICIPACIÓN DE LOS TRABAJADORES EN LOS INGRESOS DE LA EMPRESA. UN ANÁLISIS DE EFICIENCIA
}

\author{
POR \\ Juan Miguel BÁEZ MELIÁN ${ }^{1}$
}

\section{RESUMEN}

En este trabajo se analiza la relación de empleo en una empresa capitalista convencional. Su punto de partida es el carácter de información privada que tiene el desempeño del empleado. Esto hace que la relación contractual entre el empresario y el trabajador conlleve un problema relativo al esfuerzo que el trabajador dedica a la empresa. En el presente trabajo se estudian dos posibles soluciones a dicho problema: la supervisión por parte del empresario y la participación en los ingresos de la empresa por parte del trabajador, comparando ambas soluciones con la de first-best. La principal conclusión de nuestro análisis es la superioridad técnica de la empresa participativa sobre la jerárquica debido al ahorro en gastos de supervisión.

Palabras clave: relación de empleo, información oculta, supervisión, empresa democrática.

Claves Econlit: J53, J54, M51, P120

\section{THE PARTICIPATION OF THE WORKERS IN THE INCOME OF THE COMPANY. AN ANALYSIS OF EFFICIENCY}

\begin{abstract}
In this paper the employment relationship is analyzed in a conventional capitalist company. The starting point is the type of private information related to the kind of work carried out by the employee. This results in the contractual relationship between the

\footnotetext{
${ }^{1}$ Juan Miguel BÁEZ MELIÁN: Departamento de Dirección y Organización de Empresas Universidad de Zaragoza. Dirección de correo electrónico: jmbaez@unizar.es
}

REVESCO No 111 - Segundo Cuatrimestre 2013 - ISSN: 1885-8031 - www.ucm.es/info/revesco

http://dx.doi.org/10.5209/rev_REVE.2013.v111.42671

Fecha de recepción: 13/01/2012

Fecha de aceptación: 05/04/2013 
businessman and the worker creates a problem relative to the effort that the worker dedicates to the company. In this paper two possible alternative solutions are studied: supervision on the part of the businessman and the participation in company revenue on the part of the worker, comparing both solutions with the first-best. The main conclusion of our analysis is the technical superiority of the participative company over the hierarchic one due to the saving in supervising expenses.

Key words: employment relationship, hidden information, supervision, democratic firm.

\section{INTRODUCCIÓN}

El tema central de este trabajo es la relación de empleo en la empresa capitalista convencional, entendiendo esta como aquella empresa en la que existe una clara distinción entre la persona (o personas) dueña de la empresa y la mano de obra contratada por ella. Nuestra idea central es que la relación de empleo en este tipo de empresas se regula mediante un contrato de carácter incompleto, es decir, en el que no se pueden prever todas las posibles contingencias que puedan originarse en el futuro.

Estas posibles contingencias están relacionadas con diversos aspectos que afectan tanto a los intereses del propietario como al de los trabajadores. Sin embargo, nosotros nos centraremos en el carácter de información oculta que tiene el esfuerzo de los trabajadores con respecto al empresario. Obviamente esto no sería un problema si no existiera un conflicto de intereses entre el empresario y el trabajador, de no fácil solución. Al propietario le interesa que los trabajadores dediquen el máximo esfuerzo posible a la empresa con el mínimo salario (ambas cosas maximizaría el beneficio residual), y a los trabajadores les interesa todo lo contrario: mínimo esfuerzo con el máximo salario (ambas maximizaría el bienestar obtenido por su participación en la empresa).

El problema esencial consiste en que el performance de la empresa depende del desempeño que el trabajador pone en su trabajo. Sin embargo, el carácter incompleto del contrato firmado entre el trabajador y el dueño de la empresa deja abierta esta cuestión. El trabajador puede firmar que va a dedicar un determinado nivel de desempeño pero nada garantiza que vaya a cumplirlo. Dicho con otras palabras: el trabajador sabe el nivel de esfuerzo que va a dedicar a la empresa, pero eso es información privada del mismo. 
Este problema admite al menos dos tipos de soluciones. Por un lado, el empresario puede establecer un sistema de vigilancia sobre los trabajadores. Esto implicaría incurrir en costes de transacción de tipo directo. Nótese, y esta es una idea esencial de nuestro trabajo, que los recursos dedicados a este sistema de vigilancia podrían alternativamente dedicarse al propio proceso productivo (en el caso de que no fuera necesario la vigilancia), lo que aumentaría el nivel de producción. Esto nos aleja del punto de eficiencia productiva, aquel que se conseguiría si pudiéramos contratar en condiciones ideales, es decir, sin el problema de información asimétrica y el conflicto de intereses subyacente.

Otra posible solución alternativa sería la participación de los trabajadores en los ingresos de la empresa. Esto aliviaría en alguna medida el problema de incentivos planteado pero, como veremos más adelante, sólo se resolvería el problema si dicha participación es total, es decir, si la empresa fuera propiedad de los trabajadores.

En este trabajo se analizan estas dos soluciones, comparándolas con la de first best, con el objetivo de comprobar cuál de ellas es más eficiente. En el siguiente apartado haremos un breve repaso a la literatura sobre la transacción empresario/trabajador. En el tercer capítulo planteamos la solución socialmente eficiente (first best), es decir, aquel punto en donde el bienestar conjunto de ambos agentes es el máximo posible. El siguiente apartado lo dedicamos a la solución de vigilancia o supervisión por parte del empresario, poniendo el énfasis en el coste que supone para el empresario (y para la sociedad) la puesta en marcha del sistema de vigilancia. En el quinto epígrafe abordamos la solución alternativa: la participación de los trabajadores en los ingresos de la empresa, es decir, la solución no jerárquica. Nuestra idea es que dicha participación en los ingresos viene a ser una buena aproximación a cualquiera de las tres principales formas participativas: en los beneficios, en la gestión y en la propiedad de la empresa. En el capítulo sexto planteamos a modo de resumen un ejemplo numérico con el que intentamos clarificar nuestro planteamiento básico. En el capítulo séptimo hacemos una breve discusión sobre el tema a partir del análisis hecho en los epígrafes anteriores. Por último, terminamos con el habitual apartado de conclusiones.

En definitiva, lo que queremos abordar en este trabajo es comprobar si la supervisión por parte del empresario propietario sobre los trabajadores de la empresa es socialmente eficiente. O si, por el contario, la puesta en marcha de cualquier forma participativa podría generar ganancias en términos de bienestar. Pensamos que el debate implícito que estamos considerando es interesante en sí mismo, ya que aborda el carácter más o menos democrático 
del mundo laboral, es decir, de una buena parte de la vida de las personas adultas. Por otra parte, es una temática que está despertando mucho debate en los últimos años. Véase, por ejemplo, las múltiples propuestas de participación laboral por parte de las instituciones europeas. En general, la razón de dicha participación es doble: la búsqueda de una mayor eficiencia mediante una mayor implicación del trabajador en la empresa y la implantación de los valores democráticos en el mundo empresarial. En una buena parte de la literatura ambos tipos de objetivos se ven antagónicos. En este trabajo se defiende que son complementarios.

\section{LA RELACIÓN CONTRACTUAL EMPRESARIO-TRABAJADOR}

Dentro del ámbito de la Ciencia Económica, el análisis de la relación empresario/trabajador es quizás una de las cuestiones más polémicas $\mathrm{y}$, por tanto, donde existe una amplia variedad de opiniones y posturas enfrentadas. Es por ello que el intento de resumir todas estas opiniones en el reducido espacio de un epígrafe de un artículo resulta una tarea muy complicada. Para empezar hay que decir que dicha relación es sustancialmente diferente a la que existía entre el señor y el siervo en un régimen feudal, o entre el amo y el esclavo en el sistema esclavista. Por otra parte, desde el surgimiento del capitalismo, cuando comienza la interacción entro los dueños de los medios de producción (empresarios) y las personas que trabajan para ellos (trabajadores), la institución empresarial ha pasado por diferentes etapas y formas organizativas, lo que complica aún más la tarea mencionada.

Uno de los problemas más importantes que genera esta evolución organizativa (y la tecnológica correspondiente) es que los sistemas normativos y de aculturación evolucionan de forma bastante más lenta (Ben-Ner y Putterman, 1999). Las últimas generaciones de la sociedad industrializada ha sufrido una transformación que resulta crucial: hasta hace unas cuantas décadas las personas vivían en una sola localidad, producían la mayoría de los productos que necesitaban e interactuaban con un reducido número de personas a lo largo de toda su vida en una estructura económico-social bastante rígida. En la actualidad, la mayoría de nosotros dependemos de la venta de las mercancías o servicios en cuya producción participamos, trabajamos para varias empresas y en varias localidades durante nuestra vida. La menor interacción con el mismo grupo de personas dificulta el desarrollo de lealtades y afecciones, lo que resulta un obstáculo para poder beneficiarse de un comportamiento recíproco (Ben-Ner y Putterman, 1999). 
Otra dificultad añadida es que una buena parte de la teoría económica ha tratado a la empresa como una "caja negra" en la que se llevan a cabo una serie de intercambios bajo la autoridad del empresario (a diferencia de los intercambios en el mercado, donde, a priori, no existe jerarquía entre los oferentes y demandantes), sin preocuparse mucho de la naturaleza de dichos intercambios. Sin embargo, para otra buena parte de la teoría convencional dicha autoridad simplemente no existe. Véase, por ejemplo, la siguiente frase: "un empleador no tiene sobre el empleado más autoridad que un cliente sobre su proveedor" (Arruñada, 1990).

La visión que se tiene sobre la interacción empresario/trabajador está muy relacionada con la visión de la propia existencia de la empresa. Esta puede deberse a la existencia de costes de transacción (Coase, 1937), al ahorro de los costes de supervisión (Alchiam y Demsetz, 1972) o a la explotación de los trabajadores por parte de los propietarios capitalistas (Gintis, 1976).

Si consideramos al empresario como un nexo contractual entre los diferentes factores que integran la empresa (Arruñada, 1990), esta en sí misma carece de objetivos propios. Son las personas que la componen las que tienen objetivos a maximizar, por lo que pueden surgir conflictos entre ellos. Uno de estos posibles conflictos es precisamente el existente entre el empleador propietario y el trabajador no propietario. La clave para entender este conflicto está en la idea que tengamos sobre la naturaleza humana. En este sentido, podemos distinguir dos líneas de pensamiento sustancialmente diferentes.

Por un lado, tenemos una línea de pensamiento que desarrolla el punto de vista de la teoría más convencional, cuyos principales autores son Coase (1937), Alchian y Demsetz (1972), Holstrom (1982) y Jensen y Meclinkg (1994). Para estos autores la reconciliación entre los intereses particulares de los agentes y los intereses colectivos tiene lugar mediante el predominio de los primeros sobre los segundos. Se llega incluso a negar la existencia de los intereses colectivos: "las organizaciones o grupos de individuos no tienen preferencias; sólo los individuos en sí mismos pueden tener preferencias” (Jensen y Meclinkg, 1994).

El principal desarrollo de esta visión es la Teoría de la Agencia que es usada para analizar la relación entre el empresario y el trabajador. Según esta teoría el empresario (principal) debe vigilar al trabajador (agente) para que éste actúe siguiendo los intereses de aquél, aunque también se reconoce que la relación de agencia puede tener la dirección inversa, es decir, que el trabajador puede ejercer de principal, especialmente cuando el 
trabajador debe invertir en capital humano específico a la empresa (Arruñada, 1990). En cualquier caso, para esta línea de pensamiento la vigilancia implica un consumo de recursos que no pueden dedicar a la producción pero, a menos que se cambiara la condición humana, esta sería aún menor si no existiera dicha vigilancia. La cuestión de quién soporta estos costes dependerá de si el escaqueo del trabajador es anticipado por el empresario, o no. En el primer caso el coste será soportado entre ambas partes, mientras que si el escaqueo no es anticipado por el empresario será éste quien soporte el mismo.

La segunda línea de pensamiento tiene en cuenta en mayor medida los intereses colectivos. En ella podemos destacar, entre otros, los trabajos de Shapiro y Stiglitz (1984), Bowles (1985), Bowles y Gintis (1998) y Ben-Ner y Putterman (1999). Una idea central de estos autores es la profundización de la democracia como una forma de generar bienestar social. En este sentido, la eliminación de las formas jerárquicas en la empresa o, en otras palabras, el fomento de la empresa participativa es una forma de profundización democrática. Básicamente, existen tres formas de participación de los trabajadores: en la propiedad, en los beneficios y en la gestión de la empresa. Las tres igual de importantes para generar bienestar, aunque pensamos que la participación en la gestión resulta crucial para que las otras dos resulten efectivas (Báez, 2011).

La superioridad técnica de la empresa democrática es defendida por Bowles y Gintis (1993 y 1998), basándose en tres razones. En primer lugar, porque siendo los trabajadores los únicos beneficiarios del beneficio residual es razonable pensar que estarían dispuestos a aportar un mayor esfuerzo (efecto participación). En segundo lugar, porque los trabajadores tienen incentivos para el monitoreo mutuo, por lo que la empresa democrática podría desarrollar una estructura de control más efectiva (efecto monitoreo mutuo). Y por último, está el hecho de que el salario ofrecido en la empresa capitalista convencional es demasiado bajo, por lo que existe un rango de estructura de propiedad y esquemas de pagos por parte de la empresa democrática que le permitiría superar esta fuente de ineficiencia (efecto incentivo de salario).

Otra idea central en esta línea de pensamiento es el rechazo al concepto de "homo economicus", es decir, al agente perfectamente racional. "No existe ninguna base científica sobre la que asumir que el bienestar o control propio sobre recursos es la preocupación exclusiva e inmutable de las personas" (Ben-Ner y Putterman, 1999). Para estos autores el concepto de racionalidad limitada ha sido un importante avance, pero insuficiente. Las reglas 
de juego y las instituciones que las generan han de tenerse en cuenta si se pretende un análisis más realista.

El análisis de casos reales de empresas democráticas han proporcionado pistas de cómo se modifican las relaciones laborales cuando la figura del empresario supervisor desaparece. Por ejemplo, Deledicque et, al. (2005) realizaron un estudio de una empresa controlada por los trabajadores en Argentina. Según estos autores, básicamente se mantuvo la misma estructura organizativa, aunque se eliminaron los cargos de supervisión y control y se redujo el trabajo administrativo. Las nuevas reglas de trabajo no fueron fáciles de implantar, manteniéndose la tensión entre actitudes cooperativas y no-cooperativas, basándose las nuevas relaciones en una mayor igualdad y confianza.

\section{EL MODELO DE FIRST BEST}

Consideramos en este apartado una transacción entre un empresario y un trabajador en la que no existe el problema de información oculta ya comentado. En este caso no hacen falta ni el gasto en vigilancia por parte del empresario ni la participación en los ingresos por parte del trabajador. El bienestar de ambos agentes se mide mediante las funciones de beneficio y utilidad respectivamente. El beneficio del empresario viene dado por la siguiente función:

$$
\mathrm{B}^{\mathrm{o}}=\mathrm{I}-\mathrm{C}=\mathrm{p} \cdot \mathrm{q}(\mathrm{e},)-\mathrm{w}
$$

donde $\mathrm{p}$ es el precio del bien producido, q es la cantidad producida (que depende a su vez del esfuerzo dedicado por parte del trabajador, e, siendo q'(e) > 0 y q'(e) < 0) y w es el salario pagado al trabajador (estamos suponiendo que el resto de los costes, o no existen, o son fijos).

Por su parte, la función de utilidad del trabajador podría tener la siguiente forma:

$$
\mathrm{U}=\mathrm{w}-\mathrm{c}(\mathrm{e})
$$

donde c(e) es el coste del esfuerzo (c'(e) $>0$ y c', (e) $>0)$.

En la figura 1 representamos el equilibrio del trabajador que recibe un salario fijo $\left(\mathrm{w}_{0}\right)$ a cambio del esfuerzo que dedica a la empresa, aunque dicho esfuerzo representa un coste para él. El área representada por el rectángulo abcd incluye todos los posibles puntos de equilibrio. Esta área está acotada por el salario máximo $\left(\mathrm{w}_{\max }\right)$, dado por las posibilidades de supervivencia de la empresa, el salario mínimo $\left(\mathrm{w}_{\mathrm{min}}\right)$, dado por la legislación laboral vigente, 
el esfuerzo máximo $\left(\mathrm{e}_{\mathrm{max}}\right)$, limitada por las condiciones físicas del trabajador, y el esfuerzo mínimo $\left(\mathrm{e}_{\min }\right)$, dado por la costumbre socialmente aceptada.

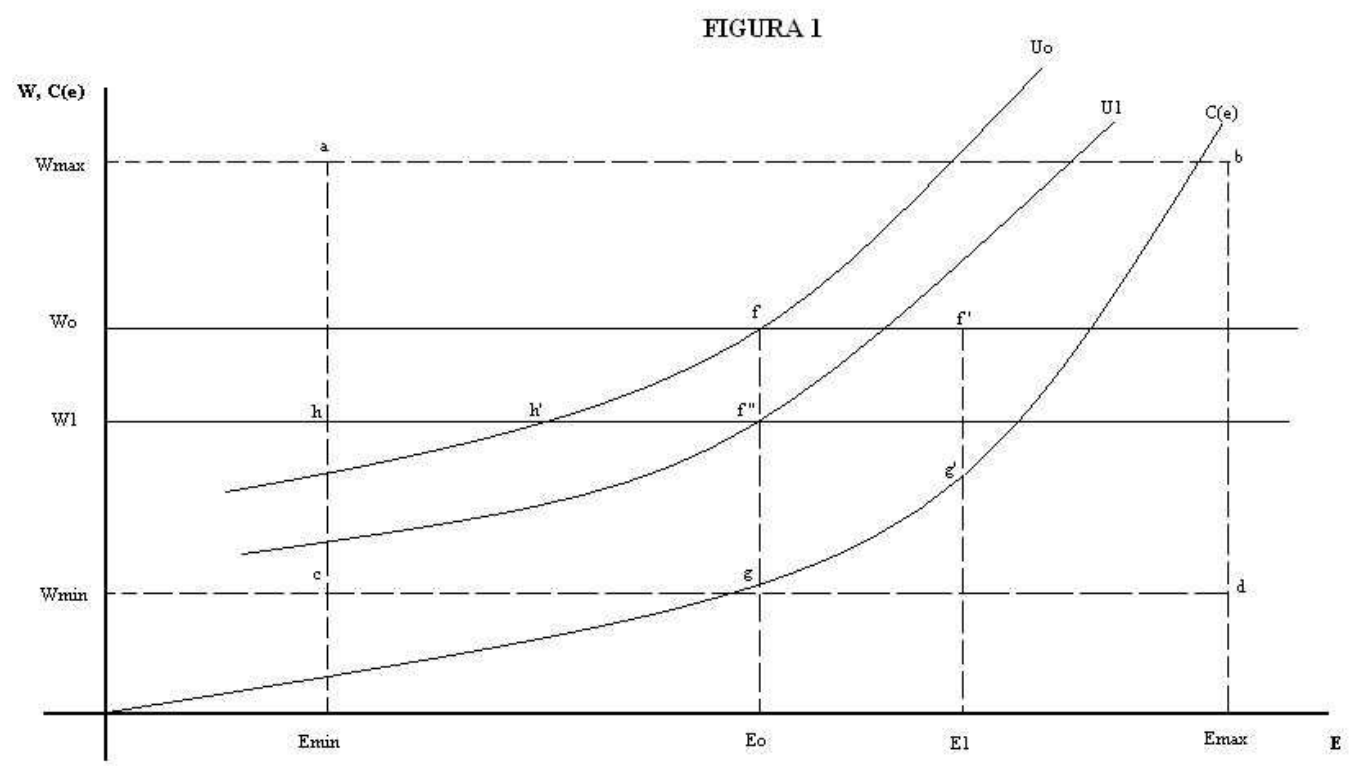

Cualquier punto perteneciente a dicho rectángulo podría ser de equilibrio. El trabajador tratará de acercarse lo más posible al punto a (máximo salario con mínimo esfuerzo), alejándose del punto d (mínimo salario con máximo esfuerzo). El salario, dentro de los límites establecidos, será mayor o menor en función del poder negociador de ambas partes. La cantidad de esfuerzo dependerá de la costumbre (ya que en este modelo no existe problema de información oculta). Suponiendo un poder negociador igual y que el esfuerzo socialmente aceptado (para ese salario) fuese $\mathrm{e}_{0}$, el equilibrio se alcanzaría en el punto $\mathrm{f}\left(\mathrm{w}_{0}=\right.$ $\left.\left(\mathrm{W}_{\max }+\mathrm{W}_{\min }\right) / 2\right)$. Por construcción la distancia fg representa el nivel de utilidad del trabajador, superior a f'g', el nivel de utilidad de reserva (representada por el nivel de bienestar que podría obtener en el desempleo o en otro trabajo alternativo). La utilidad obtenida en el punto f es la misma para toda la curva de indiferencia que pasa por dicho punto (por construcción dicha curva tiene la misma curvatura que la función del esfuerzo c(e)).

Por definición, este es el punto de eficiencia social, que podría también obtenerse maximizando en la función de bienestar social:

$$
\mathrm{FBS}=\mathrm{U}+\mathrm{B}^{\mathrm{o}}=\mathrm{w}-\mathrm{c}(\mathrm{e})+\mathrm{pq}(\mathrm{e})-\mathrm{w}=\mathrm{pq}(\mathrm{e})-\mathrm{c}(\mathrm{e})
$$


La cantidad de esfuerzo que maximiza dicha función sería la que cumpliera la siguiente igualdad:

$$
\mathrm{pq}^{\prime}(\mathrm{e})=\mathrm{c}^{\prime}(\mathrm{e})
$$

es decir, donde el valor de la productividad marginal del esfuerzo (decreciente) sea igual a su coste marginal (creciente). Para un esfuerzo inferior el valor de la productividad marginal del esfuerzo sería superior a su coste marginal, por lo que se dejarían de obtener las ganancias de las próximas unidades de esfuerzo. Para un esfuerzo superior al obtenido por la igualdad [4] el coste de las últimas unidades aportadas estaría por encima del valor producida por ellas. Un ejemplo del primer tipo de ineficiencia puede verse gráficamente en la figura 2 . Si el esfuerzo aportado por el trabajador es, por ejemplo, $\mathrm{e}_{1}$, por debajo del nivel de eficiencia, $\mathrm{e}_{0}$, la ineficiencia sería igual al área abc de la figura.

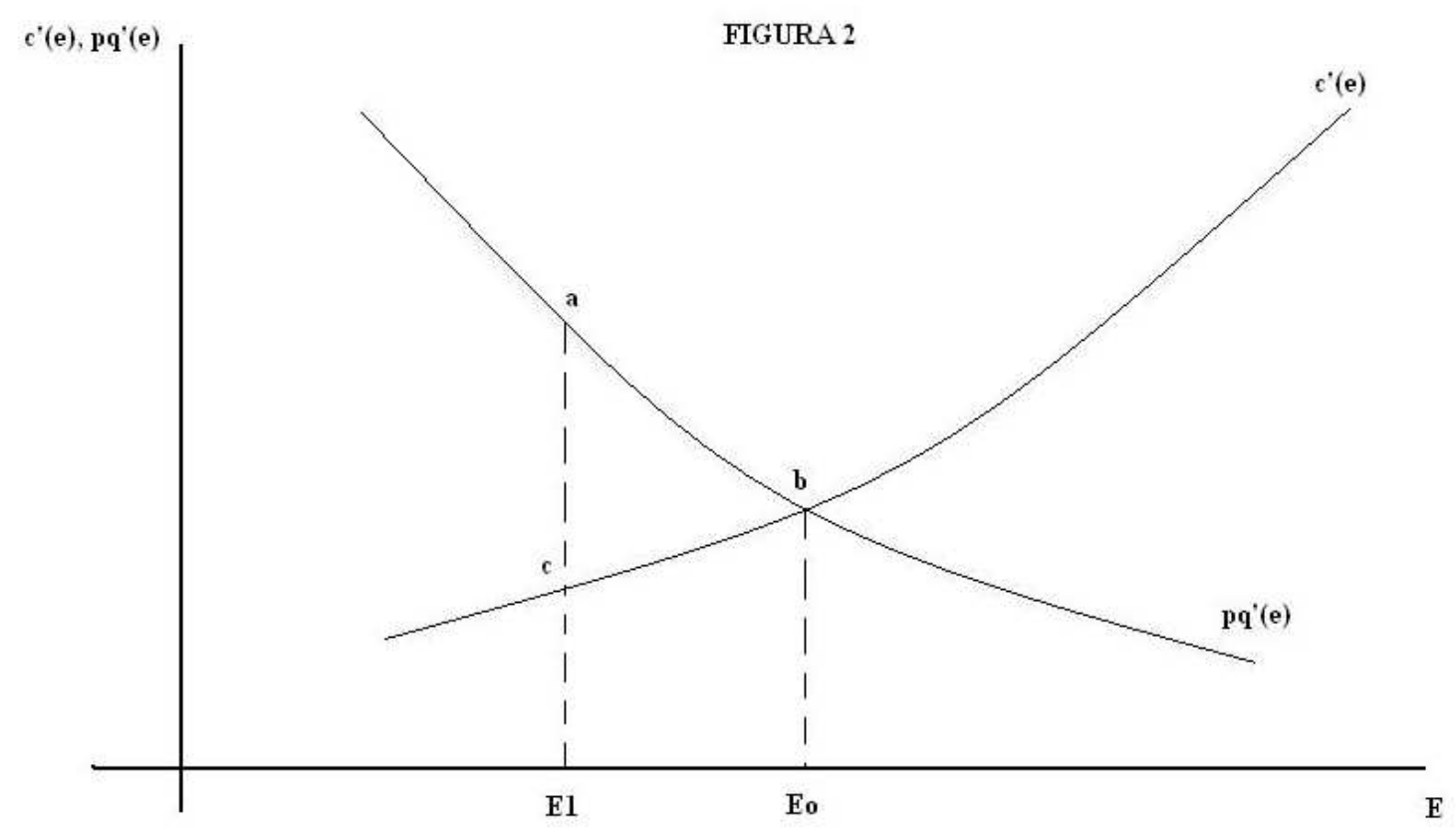

Un aumento, por ejemplo, del precio del producto (p) desplazaría hacia arriba la línea pq'(e), por lo que el nivel eficiente de esfuerzo también se incrementaría. Este aumento sería tanto mayor cuanto más horizontal sea la curva c'(e), es decir, cuanto menos costoso sea el esfuerzo. En el gráfico 1 dicho aumento se manifiesta en un desplazamiento hacia arriba de w, es decir, mediante un incremento del salario, única vía que tiene el empresario para lograr un incremento en la producción. 


\section{EL MODELO CON VIGILANCIA POR PARTE DEL EMPRESARIO}

En este modelo el empresario introduce un sistema de supervisión que supone un coste para él. Por tanto, ahora la función de beneficios de la empresa tiene la siguiente forma:

$$
\mathrm{B}^{\mathrm{o}}=\mathrm{I}-\mathrm{C}=\mathrm{p} \cdot \mathrm{q}(\mathrm{e})-\mathrm{w}-\mathrm{v}
$$

siendo v el gasto en vigilancia por parte del empresario. Sin embargo, la función de utilidad del trabajador sigue siendo la vista anteriormente [2].

En este modelo los recursos que el empresario dedica a la vigilancia del trabajador tienen una doble lectura. Por un lado, generan mayores ingresos, debido al mayor esfuerzo por parte del trabajador pero, pero por otro lado y como ya hemos comentado, constituyen un coste para él.

Por su parte, el trabajador dedicará el esfuerzo suficiente para evitar represalias que, para simplificar, podemos considerar que consisten básicamente en el despido. Esto no supondría una amenaza si no fuera por el alto ratio de desempleo que caracteriza a la mayoría de las economías. La pregunta inmediata de por qué no bajan los salarios para vaciar los mercados de trabajo tiene mucho que ver con el problema de información que se discute en este trabajo. Para conocer mejor el papel que juega el desempleo en este sentido recomendamos la lectura del trabajo de Shapio y Stiglitz (1984). Este papel esencial del desempleo también es defendido en Bowles (1985; p. 17).

Por tanto, la cantidad de esfuerzo aportada por el trabajador dependerá crecientemente de las posibilidades de que lo cojan escaqueándose, es decir, de los recursos que el empresario dedique a la vigilancia. Esto justifica, obviamente, el carácter creciente del output (y, por tanto, del ingreso) con la cantidad de recursos dedicados a la vigilancia. Sin embargo, como ya hemos dicho, estos recursos también significan un coste para el empresario, por lo que la relación entre costes de vigilancia y beneficio no es del todo clara. Dependerá de la relación que exista entre su efecto positivo (mayor esfuerzo por parte del trabajador) y su efecto negativo (coste de los recursos).

¿Cómo se modificaría el equilibrio con respecto a la situación de first best anterior? La cantidad de esfuerzo empleado dependerá ahora de la efectividad del sistema de vigilancia. Si esta fuese nula el trabajador maximizaría su bienestar en $\mathrm{e}_{\min }$ (punto $\mathrm{h}$ de la figura 1), mejorando por tanto su bienestar. En el otro extremo, cuando la vigilancia es totalmente 
efectiva, la cantidad de esfuerzo aportada por el trabajador podría incluso aumentar (siendo superior a $\mathrm{e}_{0}$ ) por lo que su bienestar disminuiría.

El nuevo equilibrio puede ser de tres tipos, dependiendo de la cantidad de esfuerzo aportada y, por tanto, de qué agente soporta la pérdida de eficiencia. Si suponemos que el esfuerzo permanece constante, el beneficio tampoco variará, ya que los recursos para establecer el sistema de vigilancia se han obtenido reduciendo el salario. Es decir, el menor salario se verá exactamente compensado por el miedo al despido. El equilibrio en la figura 1 se desplaza desde $\mathrm{f} \mathrm{a} \mathrm{f}$ ', situándose el trabajador en una curva de indiferencia inferior $\left(\mathrm{U}_{1}<\right.$ $\mathrm{U}_{0} \mathrm{y}_{0}-\mathrm{w}_{1}=\mathrm{v}$ ). Esta pérdida de bienestar por parte del trabajador representa el coste de transacción por contratar en condiciones imperfectas, es decir, con información oculta con respecto al esfuerzo. El coste de transacción es en este caso de tipo directo y es soportado en su totalidad por el trabajador.

Ahora bien, si el esfuerzo aumenta, al coste directo de la vigilancia habría que añadir la ineficiencia provocada por un esfuerzo superior a la cantidad eficiente (coste indirecto). Téngase en cuenta que esta no ha variado, dado el carácter constante del coste de vigilancia. El mayor esfuerzo hace que aumenten la producción y, por tanto, el beneficio. Es decir, el trabajador sigue soportando la totalidad del coste de la ineficiencia, pero el mismo tiene ahora tres partes: el coste directo de la vigilancia, el coste indirecto de no aplicar la cantidad de esfuerzo eficiente y el trasvase de bienestar desde el trabajador al empresario. Las dos primeras hacen referencia al problema de información asimétrica respecto a la cantidad de esfuerzo aportada, y la última es consecuencia del conflicto de intereses existente entre el empresario y el trabajador.

La segunda posibilidad es que la pérdida de eficiencia sea soportada por ambos agentes. Para que esto ocurra el nuevo equilibrio deberá situarse a la izquierda de f', pero a la derecha de h'. Es fácil comprobar que los dos agentes empeoran con respecto a la situación de first best.

La tercera y última de las posibilidades es que la ineficiencia sea soportada en su totalidad por el empresario. Para ello el nuevo equilibrio se debe situar en h' o a su izquierda. En el primer caso el bienestar del trabajador permanecerá constante, pero en el segundo habrá un trasvase de bienestar desde el empresario al trabajador. Es importante destacar que la situación del nuevo equilibrio depende crucialmente de la eficacia del sistema de vigilancia. 
Únicamente cuando el esfuerzo aumenta por encima del nivel eficiente, o disminuye por debajo del punto h', el conflicto de intereses entre ambas partes se manifiesta más claramente. En los puntos intermedios tanto el empresario como el trabajador obtienen pérdidas, aunque la distribución de las mismas dependerá del esfuerzo final, es decir, de la eficacia del sistema de vigilancia. En cualquier caso, el punto donde el esfuerzo permanece constante con respecto a la situación de first best (punto f'), puede calificarse como de second best en el modelo con vigilancia. En dicho punto la ineficiencia es provocada exclusivamente por el coste directo del sistema de vigilancia.

Vemos ahora con más nitidez la doble lectura ya comentada de los recursos dedicados a la vigilancia. Una positiva: evitan el escaqueo del trabajador, y otra negativa: son la consecuencia de un problema de asimetría de información sobre el esfuerzo del trabajador. Su existencia es la manifestación de un problema o, en sentido más pragmático, es la "solución" al problema. Pero esta solución, como hemos visto, genera una pérdida de eficiencia imposible de evitar a menos que el sistema de vigilancia sea de coste cero.

También sería interesante considerar el proceso inverso, es decir, partiendo una situación con vigilancia el empresario reduce $\mathrm{v}$ en la misma magnitud en la que aumenta $\mathrm{w}$. Para simplificar podemos suponer que ello implica la desaparición de la vigilancia. ¿Aumentaría la eficiencia? Con ayuda de nuevo del gráfico 1 podemos observar que, salvo que la reducción del esfuerzo fuera considerable (es decir, que exista un importante problema de información con respecto al esfuerzo), la pregunta anterior sólo admite una respuesta afirmativa. Parece razonable concluir que si dicho trasvase va acompañado de un cambio cultural en la organización dirigido a fomentar la confianza en la misma, tanto el beneficio del empresario como el bienestar del trabajador aumentarían, por lo que habría una ganancia de eficiencia. Esta conclusión coincide con la afirmación de Bowles y Gintis (1998) de que una de las razones de la superioridad técnica de la empresa democrática con respecto a la empresa convencional capitalista es que el sistema de disciplina de esta última es técnicamente ineficiente, en el sentido de que usa demasiados recursos en el control de esfuerzo e insuficientes en los incentivos de salario. Esta idea constituye la base para considerar la otra forma de solucionar el problema de incentivos que estamos estudiando. 


\section{OTRA FORMA DE RESOLVER EL PROBLEMA: LA PARTICIPACIÓN DEL TRABAJADOR EN EL INGRESO}

Como ya hemos comentado, una posible solución, alternativa a la vigilancia, sería la participación en los ingresos obtenidos por la empresa por parte del trabajador. Si el output, y por tanto los ingresos y los beneficios, dependen de su esfuerzo, la participación en los ingresos debería reducir la magnitud del problema de incentivos planteado, ya que las ganancias del escaqueo son ahora menores. Veamos esta cuestión más formalmente. Supongamos que el empresario ofrece el siguiente salario al trabajador:

$$
w=\beta \cdot I=\beta \cdot p \cdot q(e)
$$

es decir, un salario variable en función de los ingresos de la empresa, esto es, del esfuerzo aportado por el trabajador (siendo $0<\beta<1$ ). Si el sistema de vigilancia es eliminado el trabajador elegirá aquel nivel de esfuerzo que maximice su utilidad:

$$
U=w-c(e)=\beta \cdot p \cdot q(e)-c(e)
$$

cuyo máximo se obtiene resolviendo la siguiente igualdad:

$$
\beta \cdot p^{\prime} q^{\prime}(e)=c^{\prime}(e)
$$

Por otra parte, la condición de eficiencia sigue siendo la igualdad [4], ya que la función de bienestar social sigue teniendo la expresión [3]:

$$
F B S=U+B^{o}=\beta \cdot p \cdot q(e)-c(e)+(1-\beta) \cdot p \cdot q(e)=p \cdot q(e)-c(e)
$$

De la igualdad [8] deducimos que el trabajador elegirá una cantidad de esfuerzo inferior a la eficiente, a menos que $\beta=1$, es decir, que se lleve la totalidad de los ingresos. En esta situación el trabajador sería el dueño de la empresa y se pagaría a sí mismo un salario (cooperativa) por lo que el problema de incentivos habría desaparecido. No obstante, para $\beta<$ 1 la solución de participación también genera ineficiencia, ya que en este caso el trabajador aportará un esfuerzo inferior a $\mathrm{e}_{0}$ de la figura 2. El problema de asimetría sobre la información del esfuerzo sigue existiendo aunque pueda paliarse en alguna medida a través de la participación.

En lo que respecta al empresario, le conviene un esfuerzo que maximice su función de beneficio, que tiene ahora la siguiente forma: 


$$
B^{o}=(1-\beta) \cdot p \cdot q(e)
$$

que será igual a cero tanto si $\beta=1$ como si $\beta=0$, ya que en este segundo caso el esfuerzo también sería igual a cero y por tanto $\mathrm{q}(\mathrm{e})=0$. La intuición nos dice que debe haber un máximo en $\beta=1 / 2$, es decir, cuando los ingresos se reparte igualitariamente entre ambos agentes. Obviamente esto es así porque no estamos considerando el riesgo. En otras palabras: estamos suponiendo que tanto el empresario como el trabajador son neutrales al riesgo.

Por otra parte, si consideramos que el esfuerzo es también función de $\beta$, es decir, de la participación en el ingreso por parte del trabajador, la expresión [9] también se puede escribir como sigue:

$$
B^{o}=(1-\beta) \cdot p \cdot q[e(\beta)]
$$

De cuyo máximo se puede deducir la siguiente expresión de $\beta$ :

$$
\beta=1-\mathrm{q}[\mathrm{e}(\beta)] / \delta \mathrm{q} / \delta \mathrm{e} \cdot \delta \mathrm{e} / \delta \beta
$$

lo que indica que $\beta$ será mayor cuanto menor sea el ingreso y mayores las sensibilidades del output respecto al esfuerzo y de éste respecto a $\beta$.

Pero, ¿cuál de las dos soluciones es preferible? Nuestro modelo no nos permite responder a esta pregunta categóricamente. Sin embargo, sí podemos afirmar que cuanto mayor sea el coste de la vigilancia y la participación del trabajador en el ingreso, mayor será la probabilidad de que la opción de la participación sea preferible. A diferencia de la solución con vigilancia, esta opción no tiene un punto de second best propiamente dicho, ya que, como ya hemos comentado, su punto óptimo coincide con el first best cuando $\beta=1$.

Resulta obvio suponer que la participación en los ingresos tiene como resultado un incremento del esfuerzo del trabajador, por lo que su bienestar ha aumentado (si consideramos que el sistema de vigilancia ha desaparecido y, por tanto, el mayor esfuerzo es una decisión voluntaria). Sin embargo, el efecto sobre los beneficios no es del todo claro. Por un lado, el mayor esfuerzo por parte del trabajador incrementará los ingresos. Este efecto positivo se verá reforzado por la eliminación de los costes de vigilancia. Por otro lado, sin embargo, la menor participación del trabajador en los ingresos totales reducirá sus ingresos. Sí podemos afirmar, no obstante, que cuanto mayor sea la participación del trabajador ( $\beta$ ) la opción de la participación en los ingresos resulta preferible en mayor medida. 


\section{UN EJEMPLO NUMÉRICO}

Supongamos una empresa cuya función de producción tiene la siguiente expresión:

$$
\mathrm{q}(\mathrm{e})=21 \mathrm{e}-\mathrm{e}^{2}
$$

donde e representa la cantidad de esfuerzo aportada por el único empleado. El coste que dicho esfuerzo representa para el trabajador tiene la siguiente función:

$$
c(e)=3 e+2 e^{2}
$$

Suponiendo, para simplificar, que el precio de mercado del bien producido es $\mathrm{P}=1$, las funciones de beneficio del empresario y de utilidad del trabajador son respectivamente:

$$
B^{o}=21 e-e^{2}-w ; U=w-3 e-2 e^{2}
$$

donde w es el salario fijo pagado por el empresario. Por tanto, la función de bienestar social, que nos permite el cálculo del first best es:

$$
\text { FBS }=21 e-e^{2}-3 e-2 e^{2}
$$

cuyo máximo está en e $=3$. El esfuerzo efectivo puede ser inferior o superior a este nivel de eficiencia. Por ejemplo, si e $=2.5$ se produciría una ineficiencia de 3 unidades, mientras que si e $=3.5$ la ineficiencia sería igual a 0.75 . El valor de $\mathrm{w}$ no tiene efecto alguno sobre este punto de first best, aunque sí sobre la distribución del bienestar generado en la transacción. Por ejemplo, si w $=35$ el bienestar obtenido por cada agente en el first best sería:

$$
\mathrm{B}^{\mathrm{o}}=21 \cdot 3-3^{2}-35=19 \mathrm{y} \mathrm{U}=35-3 \cdot 3-2 \cdot 3^{2}=8
$$

¿Qué ocurriría si el esfuerzo no fuera el de eficiencia? Si e $=2.5$ entonces $\mathrm{B}^{\circ}=3$ y U $=$ 21, es decir, el empresario habría perdido 16 unidades, 13 de ellas se añadirían al bienestar del trabajador pero las otras 3 se habrían perdido. En el caso de e $=3.5$ tendríamos $\mathrm{B}^{\circ}=26.25$ y U $=0$, es decir, el trabajador habría perdido sus 8 unidades de bienestar, 7.25 de ellas serían adquiridas por el empresario, pero las restantes 0.75 se habrían perdido.

Suponemos ahora que el esfuerzo del trabajador no es observable por el empresario por lo que éste decide implantar un sistema de vigilancia con un coste de v $=5$. Esta cantidad la reduce del salario, por lo que ahora $\mathrm{w}=30$. En el cuadro 1 tenemos los principales resultados de tres casos posibles de nuevo equilibrio. En el primero de ellos (punto a de la 
figura 3) el esfuerzo sigue siendo el de first best (segunda fila), por lo que el beneficio no varía, es decir, la totalidad de la ineficiencia es soportada por el trabajador. En este caso la cuantía de dicha ineficiencia es 5 , es decir, el coste de la vigilancia (coste directo). Ahora bien, en los otros dos casos (filas tercera y cuarta) a este coste directo hay que añadir la ineficiencia derivada de producir con un esfuerzo no eficiente (coste indirecto). Cuando e = 2.5 (punto b de la figura 3) existe un trasvase de bienestar desde el empresario hacia el trabajador. Dicho trasvase ocurre en sentido inverso cuando e $=3.1$ (punto c de la figura 3 ), es decir, siempre que el esfuerzo fuese superior al eficiente. Destacar que con un esfuerzo por debajo del eficiente pero por encima de 2.65 no se produciría un trasvase de bienestar desde el empresario hacia el trabajador, ya que ambos agentes perderían bienestar.

\section{Cuadro 1}

\begin{tabular}{|l|l|l|l|}
\hline $\mathbf{e}$ & $\mathbf{B}^{\mathbf{o}}$ & $\mathbf{U}$ & $\mathbf{B S}$ \\
\hline 3 & 19 & 3 & 22 \\
\hline 2.5 & 11.25 & 10 & 21.25 \\
\hline 3.1 & 20.49 & 1.48 & 21.97 \\
\hline
\end{tabular}

Supongamos ahora que, para resolver el problema de información oculta sobre el esfuerzo, el empresario ofrece al trabajador su participación en los ingresos de la empresa. Las funciones de beneficio y utilidad son ahora:

$$
B^{o}=(1-\beta) \cdot\left(21 e-e^{2}\right) \text { y } U=\beta \cdot\left(21 e-e^{2}\right)-3 e-2 e^{2}
$$

donde $0<\beta<1$. La maximización de la segunda conduce a la siguiente expresión del esfuerzo en función de $\beta$ :

$$
\mathrm{e}=(21 \beta-3) / 2(\beta+2)
$$

cuyo valor es igual 3 (esfuerzo eficiente) si $\beta=1$, es decir, si la totalidad del ingreso es recibida por el trabajador. En el cuadro 2 tenemos los principales resultados para tres posibles valores de $\beta: 0.25,0.5$ y 0.75 . Se puede apreciar que tanto el esfuerzo como el bienestar del trabajador, así como el bienestar total, son crecientes con respecto a $\beta$. Sin embargo, el beneficio tiene un máximo en $\beta=1 / 2$, dado que nuestro modelo se desarrolla en un contexto de ausencia de riesgo. 


\section{Cuadro 2}

\begin{tabular}{|l|l|l|l|l|l|}
\hline B & e & B $^{\mathbf{o}}$ & U & BS & Punto en la figura 3 \\
\hline 0.25 & 0.5 & 7.6875 & 0.5625 & 8.25 & a' \\
\hline 0.5 & 1.5 & 14.625 & 5.625 & 20.25 & b' \\
\hline 0.75 & 2.32 & 10.8344 & 14.7784 & 25.6128 & c' \\
\hline
\end{tabular}

En la figura 3 tenemos representados los puntos considerados en los cuadros 1 y 2 , además del first best. En lo que respecta a los puntos relativos a la solución con vigilancia (puntos a, b y c) podemos apreciar que se trata de un conflicto puro (Bowles y Gintis, 1998), en el sentido de que cualquier mejora en uno de los agentes implica necesariamente el empeoramiento de la otra parte. En realidad, la vigilancia es un intento de resolver dicho conflicto (el punto de first best podría ser cualquier otro donde $\mathrm{B}^{\circ}+\mathrm{U}=27$, dependiendo del valor de w), pero el establecimiento de la misma lo que hace es reducir el tamaño de la riqueza creada a repartir.

Sin embargo, en los puntos relativos a la solución de participación (puntos a', b' y c') la cuestión no es tan obvia. Entre los puntos a' y b', es decir, para aquellos donde $\beta<0.5$, se plantea un problema de coordinación puro, ya que ambos agentes pueden mejorar hasta $\beta=$ 0.5. Para una $\beta$ superior surge de nuevo el conflicto de intereses, ya que a medida que $\beta$ aumenta el trabajador mejora pero a costa de un empeoramiento del empresario.

FIGURA 3

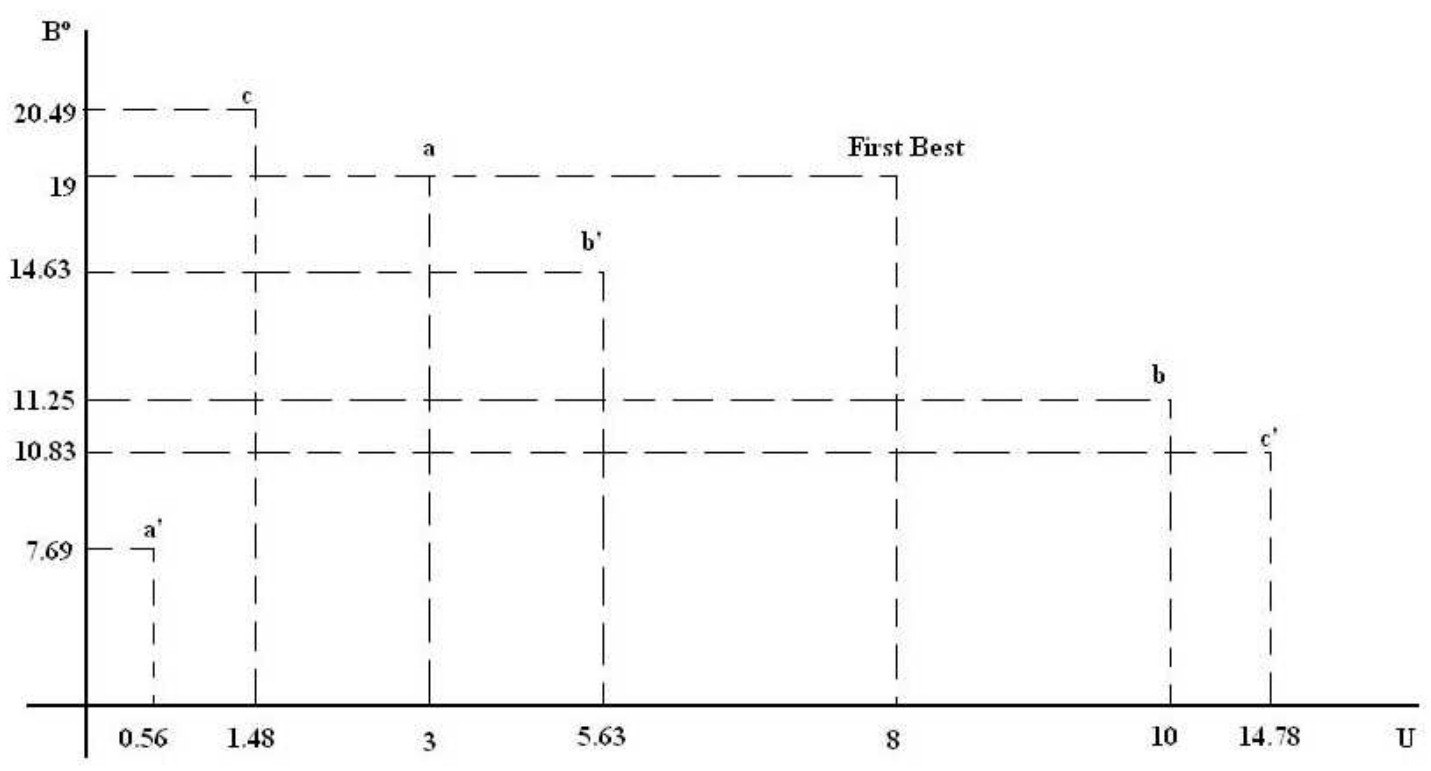


En el texto de Bowles y Gintis también se destaca estos dos ingredientes de la relación laboral: conflicto y coordinación (pp. 16-21), aunque ellos plantean el problema desde una doble perspectiva: el trabajador debe elegir entre un esfuerzo alto o bajo, pero el propietario capitalista también debe elegir entre un nivel bajo o alto de inversión en la planta. El resultado es una situación de equilibrio de Nash en la que ambos agentes aportan niveles bajos de su respectivo recurso.

\section{LA RELACIÓN LABORAL EN LA EMPRESA CAPITALISTA}

Las relaciones de poder que caracterizan a la empresa capitalista convencional distan mucho de ser democráticas. El capitalista aporta capital y el empleado aporta trabajo, pero el primero tiene poder sobre el segundo, en el sentido de que puede despedirle y puede utilizar esta capacidad y otro tipo de sanciones para obtener de él un determinado esfuerzo en beneficio propio (Bowles y Gintis, 1993). Este poder está basado en su posición favorable en el mercado. En palabras de estos autores: los empleadores se encuentran en el lado corto del mercado. Dicho de otra manera: la relativa igualdad entre oferentes y demandantes del mercado típico (al menos teóricamente) se sustituye por el poder jerárquico del empresario en el mundo de la empresa. Pensamos que los análisis sobre el desarrollo de la cultura organizacional no tienen en cuenta este aspecto fundamental, tratando igualitariamente a dos tipos de agentes que en la realidad ocupan situaciones sustancialmente opuestas.

En este trabajo hemos analizado la relación laboral que tiene lugar en el seno de este tipo de empresas. Pensamos que los hechos más relevantes que caracterizan dicha relación son dos. Por un lado, está el carácter de información oculta que tiene el esfuerzo del trabajador. El carácter incompleto del contrato de trabajo impide que el desempeño futuro del trabajador pueda ser establecido con precisión. Por otro lado, está el conflicto de intereses entre ambos agentes, en el sentido de que el interés del propietario (definido por su derecho al beneficio residual) es favorecido por su capacidad para obligar a la otra parte a actuar en forma diferente a sus deseos (Bowles, 1985). Por otra parte, las estrategias que tiene el propietario para ejercer dicho poder son costosas y, por tanto, implican una pérdida de eficiencia con respecto a la situación ideal de ausencia de conflicto.

Para centrarnos en el tema del desempeño del trabajador hemos simplificado considerablemente el modelo. Esto obviamente conlleva limitaciones. Queremos destacar cuatro. La primera de ellas es que descarga toda la tensión sobre la responsabilidad de los 
trabajadores. Son ellos los que tienen que decidir si dedican un mayor o menor esfuerzo a la empresa y el propietario se limita a pagarles más o menos, utilizando el salario o la participación como incentivos para lograr un mayor esfuerzo por parte de ellos.

Sin embargo, los trabajadores no son la única parte de la contratación que puede tener oculta información relevante. Algunas posibles actuaciones por parte del propietario son de mucho interés para los trabajadores. Por ejemplo, la inversión que va a llevar a cabo en los próximos periodos. Pero la solución a este problema tiene una complicación añadida: la vigilancia por parte del trabajador resulta más difícil, dada su condición de subordinado con respecto al empresario.

La segunda limitación es la ausencia de presión social. En sentido más amplio, hemos ignorado los aspectos culturales de la organización. Resulta difícil aceptar que alguien decida aspectos fundamentales de su comportamiento dentro de la organización (como, por ejemplo, la cantidad de esfuerzo que aporta a la misma) sin tener en cuenta el comportamiento de los otros miembros de la misma.

En tercer lugar, no estamos considerando la posición frente al riesgo de los agentes. En otras palabras, nuestro modelo se desarrolla en un contexto de neutralidad frente al riesgo. Esto afectaría sobretodo a la solución de participación en lo que respecta al reparto del output producido, aunque creemos que sin afectar sustancialmente las conclusiones del modelo.

Por último, estamos considerando sólo la participación financiera y no podemos identificar dicha participación con una mayor democracia en la empresa. Sí podemos afirmar que, según nuestro modelo, la participación financiera (que utilizamos como una aproximación a la empresa democrática) implica mayor eficiencia con respecto a la empresa capitalista convencional. Efectivamente, si $\beta=1$, es decir, si todo el output producido es propiedad de los trabajadores, se lograría la máxima eficiencia, aunque toda ella sería acaparada por los empleados. Cualquier otro punto en ambas soluciones, con vigilancia o con participación pero con $\beta<1$, seria inferior. En el primer caso por la existencia de los costes de vigilancia y en el segundo porque los incentivos que generan la participación son insuficientes. En el caso numérico visto anteriormente, si $\beta=1$ entonces $B^{\circ}=0$ y $U=27$. En términos de la figura 1 , el equilibrio se alcanzaría en la misma vertical para $\mathrm{e}_{0}$, pero en un punto por encima de $\mathrm{f}$, es decir, en una curva de indiferencia superior. 
La pregunta inmediata es la siguiente: ¿por qué no se produce una mayor expansión de la empresa democrática? Aportamos tres razones (Bowles y Gintis, 1993). La primera es que el aprendizaje de la gestión democrática lleva tiempo. A menos que las ganancias asociadas a dicha gestión sean considerables, los costes de aprendizaje y la escasez de trabajadores experimentados en este tipo de gestión pueden ser prohibitivos (restricción de capacidad democrática). En el trabajo, ya comentado, de Deledicque et, al. (2005) se puede apreciar claramente este tipo de dificultades que conlleva la creación de una empresa democrática. La segunda es la propia predominancia de la empresa capitalista convencional (restricción de medioambiente económico) y la tercera está en los requerimientos de capital y la aversión al riesgo de los propios trabajadores (restricción de desigualdad de riqueza).

Ugo Pagano (1998) aporta otra razón para la menor expansión de este tipo de empresas: la menor homogeneidad de intereses de los trabajadores con respecto a la de los propietarios accionistas. Para este autor, la redistribución de recursos hacia los trabajadores no debe implicar necesariamente la vuelta a la producción en pequeña escala. Sin embargo, es necesario tener en cuenta las dificultades que tiene el ejercicio del control por parte de los trabajadores en las grandes compañías. Una de ellas es esta falta de homogeneidad, por lo que la puesta en común y el ejercicio de decisiones colectivas resultan más complejos.

Una idea similar es la que aporta Coque (2008): en las cooperativas confluyen más actores que en las empresas de capital, y algunos de ellos asumen más de un papel. Según este autor las dificultades de gobierno en las cooperativas se deben a los problemas de determinados socios y directivos para utilizar los diferentes instrumentos en el momento y modo adecuados. Esto sucede cuando la organización cae en alguna de sus dos posibles "desviaciones": el exceso de cooperativismo, donde todo el mundo quiere decidir, con la consiguiente ralentización en el funcionamiento organizacional; o el defecto de cooperativismo, cuando determinados gestores profesionales (en alianza, o no, con algunos socios) acumulan demasiada información en detrimento del resto.

Por otra parte, las dos soluciones estudiadas en este trabajo pueden identificarse con los dos tipos de relaciones laborales en las economías desarrolladas, defendidas por Gordon (1998): sistemas conflictivos (vigilancia) y sistemas cooperativos (participación), los primeros caracterizados por un mayor gasto en supervisión y un menor gasto en incentivos de salarios. Según este autor, las economías más cooperativas pueden alcanzar ratios más altos de crecimiento de la productividad y de los salarios reales. 
En cualquier caso, no podemos identificar la democracia económica exclusivamente con las sociedades cooperativas, sino con cualquier tipo de participación empresarial, con independencia de su forma jurídica. El cooperativismo se limita a dar cobertura intelectual y jurídica a las reivindicaciones de participación (Divar y Gadea, 2007). Por otro lado, no todas las cooperativas funcionan como tales y existen empresas de capital que en la práctica funcionan como sociedades cooperativas (Coque, 2008).

Por otro lado, lo que caracteriza a este tipo de organizaciones participativas es su cultura (Hernández, J.M. et. al., 2008). Sin embargo, como afirman estas autoras, en la mayoría de las ocasiones los socios no comparten ni los valores ni los principios básicos del cooperativismo, llegando incluso algunos de ellos a demandar un funcionamiento de la organización no democrático. En definitiva, dicho funcionamiento se fundamenta en la confianza originada por las relaciones de vecindad o familiar entre socios. De ahí que una buena parte de las cooperativas tengan su origen en localidades relativamente pequeñas, en donde este tipo de relaciones personales se desarrollan con mayor facilidad.

No obstante, Díaz y Jaimez (2009) sí encuentran indicios de una cultura propia por parte de los trabajadores cooperativistas, aunque en un sentido algo diferente al esperado por ellos. En su trabajo, las mayores valoraciones por parte de los trabajadores cooperativistas se dan en las dimensiones individualista y mixta, pero no en la colectivista. Sin embargo, los propios autores defienden que dichas dimensiones no necesariamente realzan al individuo frente a la colectividad, sino que enfatizan la propia libertad del individuo. De hecho uno de los valores con mayor diferencia significativa entre los trabajadores cooperativistas y no cooperativistas es la autodirección, un valor que resalta la independencia y la libertad de actuación de la persona, aspectos que resultan básicos en el ideario cooperativista.

En el mismo sentido se sitúan los resultados del trabajo de Gargallo y Freundlich (2010). El estilo de dirección es citado por un 30.4\% de los socios entrevistados como uno de los cinco principales aspectos de la satisfacción laboral. La diferencia es significativa con respecto a los trabajadores no socios $(21 \%)$. Otras respuestas en las que las diferencias de porcentajes entre ambos tipos de trabajadores resultan significativas son los siguientes: la retribución económica, la seguridad en el empleo, la promoción, la pertenencia a la cooperativa, el respeto al medio ambiente y la mejora de la comunidad. 
En cualquier caso, el creciente desmantelamiento del sector público que se ha venido produciendo en las economías desarrolladas durante los últimos años, junto a la incapacidad del mercado para satisfacer adecuadamente las principales necesidades colectivas, han provocado un auge de organizaciones de tipo social. En dicho auge las sociedades cooperativas son las entidades de mayor significación histórica y económica (Puentes y Velasco, 2009).

De las dos principales líneas de pensamiento vistas en el apartado dos, este trabajo se enmarca en aquella que da un mayor peso a los intereses colectivos. Pensamos que el desarrollo del planteamiento más convencional, a través de las aportaciones a la Teoría de los Costes de Transacción, nos parece sumamente interesante aunque insuficiente. Su principal aportación en relación a la relación empresario-trabajador es precisamente reconocer el conflicto de intereses inherente a dicha relación. Nuestro trabajo se ha centrado en dicho conflicto y en dos formas alternativas para resolverlo: la supervisión y la participación. De la discusión de los apartados anteriores podemos deducir la superioridad técnica de la participación (en términos de eficiencia).

Por otra parte, en la solución de supervisión se ha de destacar la importancia del desempleo. Debido a ello la amenaza de despido cobra toda su relevancia, por lo que puede ser utilizada por el empresario para obligar al trabajador a aplicar un esfuerzo superior al deseado por éste. Este hecho es consecuencia de una fundamental y peculiar característica del mercado de trabajo (Gintis, 1976): los atributos personales de los agentes son una parte relevante de las transacciones. Además de las capacidades productivas del trabajador, al empresario le interesa conocer sus características personales. En definitiva, el desempleo y la supervisión son complementarios, en el sentido de que la existencia del desempleo permite a la sociedad ahorrar gastos en supervisión (Shapiro y Stiglitz, 1984). Este ahorro más que compensa las pérdidas de un nivel de empleo reducido.

\section{CONCLUSIONES}

La principal conclusión de nuestro trabajo es que la solución de supervisión es socialmente ineficiente. El problema de incentivos planteado admite otra solución que parece técnicamente superior: la participación en los ingresos. Esta conclusión coincide con la del texto ya comentado de Bowles y Gintis (1998), en el que se adopta una doble perspectiva para el análisis de la relación laboral. En palabras de estos autores, la resolución de los fallos de 
coordinación requiere con frecuencia acuerdos que son difíciles o muy costosos de llevar a cabo con las estructuras de gobernanza actualmente existentes. Un punto esencial de su argumentación, y que también es apoyado en nuestro modelo, es que criterios de eficiencia aconsejan unir en una misma persona, o grupo de personas, el derecho al uso de un medio de producción y la propiedad sobre el mismo. Recuérdese que en las dos soluciones vistas la única vía para alcanzar el first best es mediante la participación, cuando $\beta=1$, es decir, cuando la empresa es propiedad de los trabajadores. Es obvio que en este caso los costes de control desaparecen. Lo que demostramos en este trabajo es que en la empresa capitalista convencional (es decir, en la solución de supervisión) y desde el punto de vista de la eficiencia, estos costes no son compensados con una mayor producción.

\section{BIBLIOGRAFÍA}

ALCHIAN, A. Y DEMSETZ, H.: Production, Information Costs, and Economic Organization. American Economic Review, nº 62, Diciembre, 1972, p. 777-795.

ARRUÑADA, B.: Economía de la Empresa: un enfoque contractual. Barcelona: Editorial Ariel, 1990. 398 p. ISBN: 8434420481.

BÁEZ, J.M.: La participación de los trabajadores en la empresa. El caso de Irlanda. CIRIECEspaña, Revista de Economía Pública, Social y Cooperativa, no 70, 2011, p. 127-148.

BÁEZ, J.M.: Las preferencias y la conducta del cooperante recíproco en un contexto empresarial. Cuadernos de Economía y Dirección de Empresas, Vol. 15, nº 1, 2012, p. 2532.

BEN-NER, A. Y PUTTERMAN, L.: Valores e instituciones en el análisis económico. CIRIEC-España, Revista de Economía Pública, Social y Cooperativa, no 33, 1999, p. 4377.

BOWLES, S.: The Production Process in a Competitive Economy: Walrasian, NeoHobbesian, and Marxian Models. American Economic Review, Vol. 75, N. 1, March, 1985, p. 16-36.

BOWLES, S. Y GINTIS, H.: The Democratic Firm: An Agency-Theoretic Evaluation. En: BOWLES, S., H. GINTIS Y B. GUSTAFSSON (eds.). Markets and Democracy: participation, accountability and efficiency. Cambridge: Cambridge University Press, 1993, 360 p. ISBN: 978- 0521064118.

BOWLES, S. Y GINTIS, H.: Efficient redistribution: New Rules for Markets, States and Communities. En: S. BOWLES Y H. GINTIS. Recasting Egalitarianism. New Rules for 
Markets, States and Communities. New York: Erik Olin Wright, 1998, p. 3-71. ISBN: 1$85984-863-X$

COASE, R.H.: The Nature of the Firm”, Economica, Vol. 4, n 16, 1937, p. 386-405.

COQUE, J.: Puntos Fuertes y débiles de las cooperativas desde un concepto amplio de gobierno empresarial. REVESCO, Revista de Estudios Cooperativos, nº 95, 2008, p. 6593.

DELEDICQUE, L.M., M. FÉLIZ Y J. MOSER.: Recuperación de empresas por sus trabajadores y autogestión obrera. Un estudio de caso de una empresa en Argentina. CIRIEC-Revista de Economía Pública, Social y Cooperativa, nº 51, 2005, p. 51-76.

DÍAZ, F. Y JAIMEZ, M.: ¿Existe una cultura cooperativa organizacional en trabajadores cooperativistas y no cooperativistas?. REVESCO, Revista de Estudios Cooperativos, $\mathrm{n}^{\circ}$ 97, 2009, p. 44-57.

DIVAR GARTEIZ-AURRECOA, J. Y GADEA SOLER, E.: Desarrollo territorial, participación y cooperativismo. REVESCO, Revista de Estudios Cooperativos, $\mathrm{n}^{\circ}$ 92, 2007, p. 132-152.

GARGALlO, A. Y FREUNDLICH, F.: Percepciones de los socios y no socios cooperativistas sobre la satisfacción laboral. REVESCO, Revista de Estudios Cooperativos, $\mathrm{n}^{\circ} 103,2010$, p. 33-58.

GINTIS, H.: The Nature of the Labor Exchange and the Theory of Capitalist Production. Review of Radical Political Economics, Vol. 8, n² 2, 1976, p. 36-54.

GINTIS, H. Y ISHIKAWA, T.: Wages, Work Intensity, and Unemployment. Journal of the Japanese and International Economies, Vol. 1, 1987, p. 195-228.

GORDON, D.M.: Conflict and Cooperation: An Empirical Glimpse of the Imeratives of Efficiency and Redistribution. En: S. BOWLES Y H. GINTIS. Recasting Egalitarianism. New Rules for Markets, States and Communities. New York: Erik Olin Wright, 1998, p. 181-207. ISBN: $1-85984-863-\mathrm{X}$

HERNÁNDEZ, M.J., RUIZ, C. Y GARCÍA, E.: La cultura como mecanismo de gobierno y control en las organizaciones cooperativas. REVESCO, Revista de Estudios Cooperativos, $n^{\circ}$ 95, 2008, p. 94-111.

HOLMSTROM, B.: Moral Hazard in Teams. The Bell Journal of Economics, Vol. 13, 1982, p. $324-40$.

JENSEN, M.C. Y W.H. MECKLING.: The Nature of Man. Journal of Applied Corporate Finance, Vol. 7, No. 2, 1994, p. 4 - 19. 
PAGANO, U.: Redistributions of Assets and Distributions of Asymmetric Information. En: S. BOWLES Y H. GINTIS. Recasting Egalitarianism. New Rules for Markets, States and Communities. New York: Erik Olin Wright, 1998, p. 285-318. ISBN: 1-85984-863-X.

PUENTES, R. Y VELASCO, M.M.: Importancia de las sociedades cooperativas como medio para contribuir al desarrollo económico, social y medioambiental, de forma sostenible y responsable. REVESCO, Revista de Estudios Cooperativos, nº 99, 2009, p. 104-129.

SHAPIRO, C. Y STIGLITZ, J.: Unemployment as a worker discipline device. American Economic Review, Vol. 74, N. 3, 1984, p. 433-44. 